\title{
Competencia motriz, compromiso y ansiedad de las chicas en Educación Física Motor competence, commitment and anxiety in Girls in physical education classes
}

*Gurutze Luis-de Cos, **Silvia Arribas-Galarraga, **Izaskun Luis-de Cos, ** José Antonio Arruza Gabilondo

*Universidad Nacional de Educación (Ecuador), **Universidad del País Vasco (España)

\begin{abstract}
Resumen. Durante las últimas décadas la figura de la mujer parece que va adquiriendo un papel más notable en la sociedad en general y en la actividad físico-deportiva en particular, sin embargo, perduran ciertos estereotipos de género que influyen en la práctica de las chicas. El presente trabajo estudió la competencia motriz en de las adolescentes, y su relación con aspectos psico-sociales. Se analizó el nivel de competencia motriz de un grupo de chicas de Enseñanza Secundaria Obligatoria, fijando la atención sobre el compromiso en las sesiones de educación física (EF) y el nivel de ansiedad que estas sesiones les generaban. La muestra estuvo compuesta por 433 jóvenes de entre 12 y 15 años. Se empleó una metodología cuantitativa de naturaleza descriptiva y correlacional. Entre los resultados obtenidos, destacar que más del $70 \%$ de las participantes se situó en niveles medio-altos de competencia motriz, resultando que este nivel aumentaba con la edad (27.9\% con alta competencia motriz a los 12 años, y $46.5 \%$ a los 15 años). Así pues, mientras que el compromiso con las sesiones de EF, disminuía paulatinamente con la edad (en un rango de 1 a 4, las chicas de 12 años presentan $M=3.08$, y las de 15 años $M=2.82$ ), la ansiedad aumentaba ligeramente. Finalmente, se advirtió que las adolescentes que tenían mayor nivel de competencia motriz eran las más comprometidas con las clases de EF y presentaron menores niveles de ansiedad y estrés, siendo las relaciones entre las variables psico-sociales y el nivel de competencia motriz estadísticamente significativas. Se concluye que un mayor nivel de competencia motriz podría influir positivamente en los aspectos psico-sociales estudiados.
\end{abstract}

Palabras Clave: mujer, escuela, deporte, destreza, motivación.

Abstract: The present study aims to investigate the evolution of motor competence in female adolescents' development and its relationship with psycho-social aspects. To this end, the level of motor competence of a group of who attend Secondary School is analyzed, setting attention to the commitment in physical education sessions and the level of anxiety generated by these sessions. The sample is composed of 433 female young Basque people between 12 and 15 years old. A quantitative methodology of descriptive and correlational nature was used. Among the results obtained, it can be highlighted that more than $70 \%$ of the participants are placed in medium-high levels of motor competence, turning out that this level increases with age $(27.9 \%$ with high motor competence at 12 years, and $46.5 \%$ at 15 years old). Thus, while the commitment to Physical Education (PE) sessions decreases gradually with age (in a range of 1 to 4, 12-year-old girls present $M=3.08$, and 15-year-old girls $M=2.82$ ), anxiety increases slightly. Finally it is noticed that the female adolescents who have higher level of motor competence are the ones who commit more in Physical Education classes and show lower levels of anxiety and stress, being the relationships between psycho-social variables and the level of motor competence statistically significant. It is concluded that a higher level of motor competence could positively influence the psycho-social aspects studied.

Keywords: woman, school, sport, skill, motivation.

\section{Introducción}

La actividad físico-deportiva en general y la educación física (EF) en particular, son reflejo de los estereotipos de género típicos de la sociedad donde se desarrolla. Desde tiempos inmemorables, la sociedad occidental ha identificado la imagen masculina con un ser de carácter dominante, fuerte, activo; por el contrario, la imagen de la mujer se ha dibujado como sensible, débil y pasiva. Sever (2005) explica cómo el deporte masculino se relaciona con la fuerza, la potencia, la resistencia... y el deporte femenino se asocia a la expresividad, la flexibilidad, el ritmo. Esta imagen, ha mantenido durante años a la mujer alejada de actividades físicodeportivas exclusivamente destinadas a los hombres, y fueron muchos los esfuerzos que algunas personas realizaron para salvar los modelos heredados (por ejemplo: Charlotte Cooper, Kathrine Suitzer, Lili Álvarez...). Sin embargo, diferentes autores (Fontecha, 2006; Pelegrín, León, Ortega \& Gracés de Los Fayos, 2012), afirman que siguen existiendo barreras que dificultan una participación proporcionada en actividades físico-deportivas entre mujeres y hombres, y que aún hoy no se puede afirmar que la educación igualatoria

Fecha recepción: 10-04-18. Fecha de aceptación: 14-02-19 Gurutze Luis de Cos

gurutze.luis@unae.edu.ec para ambos sexos haya sido conseguida en EF (Moreno, Alonso \& Martínez, 2005). Así lo muestra recientes estudios, como el que realizado con1626 alumnos y alumnas de ESOBachillerato y en el que se analizó la práctica deportiva-recreativa en función de género revelando un mayor índice de participación en chicos, y diferencias significativas a favor de los chicos en el tipo de interacción motriz y espacio de juego (Lamoneda \& Huerta, 2017). En la misma línea, fueron estudiadas las etapas de cambio ante la práctica de actividad física en el tiempo libre en 1112 estudiantes de entre 12 y 15 años, constando que los chicos son más activos que las chicas (Gallegos-Sánchez, Ruiz-Juan, Villarreal-Angeles \& Rivera, 2019).

El término «competencia» fue definido por White en 1959 como la capacidad de un organismo para interactuar con su medio de forma eficaz. Al relacionar la competencia con el ámbito de la EF, surge el concepto de «Competencia Motriz» (Ruiz, 1995; 2014). La competencia motriz ha sido estudiado desde diferentes perspectivas pero todas tienen en común que ven como objetivo principal la capacidad de la persona para llevar a cabo tareas motrices complejas para ser eficaz y eficiente en su medio (Ruiz-Pérez, Rioja-Collado, GrauperaSanz, Palomo-Nieto \& García-Gil, 2015b). El desarrollo de la competencia motriz implica una conexión de diferentes dimensiones:1) la afectiva-social, que hace referencia al con- 
junto de decisiones y emociones que se adoptan en el proceso de aprendizaje (Ariolfo, 2010);2) la dimensión perceptivo-cognitiva, que se puede describir como la implicación de una serie de mecanismos y procesos que activan operaciones de carácter cognitivo y perceptivo y que evolucionan durante la vida influyendo notablemente en el desarrollo motor (Ruiz \& Arruza, 2005); y 3)la dimensión neuromotora, asociada a las acciones motrices. Se puede decir, que la competencia motriz está compuesta por las diferentes destrezas que disponemos para realizar movimientos y solucionar problemas motores que se den tanto en las tareas cotidianas como dentro de la práctica deportiva. Teniendo en cuenta esta premisa, diversos autores consideran que la principal finalidad de la EF es el desarrollo de la competencia motriz (Connolly, 1980; Ruiz 2014; Ruiz \& Graupera, 2005; Ruiz \& Linaza, 2013). En esa línea, y para determinar una gradación en el nivel de competencia, un estudio global realizado en las comunidades de Aragón, Cataluña, País Vasco y Andorra - Comunidad de Trabajo de los Pirineos- con adolescentes, organizó los datos en torno a tres niveles de competencia motriz: bajo nivel, nivel medio y alto nivel de competencia motriz (Arruza, Irazusta \& Urrutia-Gutierrez, 2011). Dichos niveles resultaron de establecer percentiles según edad y sexo de los participantes. Se asociaron a la baja competencia todas aquellas puntuaciones que se encontraban por debajo del percentil 33, media competencia motriz las que se encontraban entre el percentil 33 y 63 , y alta competencia las que estaban por encima del percentil 63. Los jóvenes presentaron nivel medio y alto de competencia motriz, siendo las chicas quienes presentaron los valores más discretos. Por otro lado, el estudio realizado por Cantó, García, Soto, Guillamon \& Villalba, (2015) demostró que la autopercepción de competencia motriz es una variable que se relaciona positivamente con los niveles globales de actividad física habitual, y concluye que estos resultados apuntan la necesidad de orientar la promoción de la práctica físico-deportiva hacia modelos de desarrollo de la competencia motriz. Sin embargo, también parecen existir diferencias en la percepción de competencia en función del sexo. UrrutiaGutierrez, Irazusta-Adarraga, Balerdi-Iztueta, GonzálezRodríguez \& Arruza-Gabilondo (2013), estudiaron a 366 sujetos de 13 a 16 años siendo que los chicos presentaron valores más altos en percepción de competencia motriz $(55,7 \%$ nivel medio de competencia) que las chicas ( $56 \%$ se percibe con un bajo nivel de competencia).

En el proceso de adquisición de competencias es fundamental que el alumnado sea consciente de las acciones y habilidades que requieren la solución de problemas, pudiendo de esta manera desarrollar, aplicar y reflexionar sobre sus resultados, y así completar el ciclo del logro de las competencias y poder transferirlas a nuevas situaciones que se planteen. Para que este proceso sea posible es necesaria la disposición activa y responsable del alumnado hacia el proceso de aprendizaje. En este sentido, Yoo (2015) en un estudio realizado con 592 jóvenes de educación secundaria halló que la motivación autónoma media positivamente con la competencia percibida y con el compromiso conductual, y añade la importancia de la emoción positiva como potenciador de los procesos de motivación. Asimismo, existen estudios que afirman que los chicos se consideran más capacitados para realizar actividades físicas que las chicas, dedicando más tiempo a la práctica fiìsico-deportiva y consecuentemente muestran un perfil de autopercepción más positivo que ellas (Hellì̀n, Moreno \& Rodrì̀guez, 2006). Otros estudios (Cairney, Kwan, Velduizen, Hay, Bray \& Faught, 2012) que asocian positivamente el disfrute en EF con la competencia percibida, indican que las niñas son quienes presentan menores niveles de disfrute.

Podría decirse, que el compromiso es el grado en el que el alumnado se implica y conecta activamente con el aprendizaje (Rigo, 2014; Rigo \& Donolo, 2014).Así pues, se entiende el compromiso como un concepto multidimensional integrado por componentes cognitivos, conductuales y afectivos (Arguedas, 2010; Frederisck, Blumenfeld \& Paris, 2004), estando relacionados directamente con la calidad y la cantidad de participación del alumnado en la asignatura. Por tanto, para abordar el aprendizaje, resulta necesario estudiar los factores que influyen sobre su diversión y su sentimiento de competencia (Stuntz \& Weiss, 2009). Asimismo, el compromiso se ha considerado un aspecto determinante en la implicación en las clases de EF, puesto que estudios anteriores han comprobado que el compromiso en el aprendizaje es fundamental en la participación activa de las y los adolescentes (Astin, 1999; Coterón, Franco, Pérez-Tejero \& Sampedro, 2013; Lima, Saavedra, Fernandes, Lazuras, \& Barkoukis, 2015; Prieto, 2016; Rigo \& Donolo, 2014). Un estudio realizado con 800 participantes (413 Chicos y 387 Chicas) de entre 11 y 17 años, constató gran falta de compromiso con la actividad físico-deportiva al verificar un alarmante abandono y disminución de los niveles de práctica a lo largo de la etapa de la adolescencia, acentuándose esta evidencia en mayor medida en el segundo ciclo educativo y en las chicas frente a los chicos (Navarro-Valdivielso, Ojeda-García, Navarro-Hernández, López-López, Brito-Ojeda\& Ruíz-Caballero, 2012). Sin embargo, un estudio realizado con chicas jóvenes concluyó que las adolescentes que realizaban actividad físico-deportiva con mayor frecuencia eran quienes obtenían puntuaciones más altas en competencia motriz, y presentaban mayor intención de praìctica futura (ArribasGalarraga, Luis-de Cos, Luis-de Cos \& Saies, 2018).

Por otro lado, mediante las teorías de metas de logro (Ames, 1987) y la teoría de la autodeterminación (Deci \& Ryan, 1985), se ha comprobado que la percepción de un clima tarea o maestría se relaciona positivamente con actitudes positivas hacia la asignatura, niveles altos de compromiso y motivación intrínseca (Cera, Almagro, Conde \& SáenzLópez, 2015; Coterón, et al., 2013; Gutiérrez, 2014; MéndezGiménez, Fernández-Rio \& Cecchini-Estrada, 2013; Moreno \& Cervelló, 2010; Morente, Zagalaz, Molero \& Carrillo, 2012). En esta línea, un estudio realizado con 1298 estudiantes de Educación Secundaria mostró que las chicas se sentían más cómodas en un clima de aprendizaje (Granado-Gallegos \& Baena-Extremera, 2014). Asimismo, otro estudio estableció una relación positiva entre la percepción de un clima tarea y el compromiso (Torregrosa, Sousa, Viladrich, Villamarín \& Cruz, 2008; Torregrosa, Viladrich, Ramis, Azócar, Latinjak \& Cruz, 2011).

Entre los aspectos que estudian la motivación del individuo en relación a la participación en las clases de EF, la ansiedad ante el error y el fracaso se constató como aspecto 
que pudiera determinar el rendimiento (Duda \& Hall, 2001).Varios estudios encontraron que la ansiedad correlacionaba positivamente con la percepción de un clima ego y negativamente con la percepción de un clima tarea (Cecchini, González, López \& Brustad, R. J., 2005; Smith, Smoll \& Cumming, 2007). Asimismo, diferentes estudios han relacionado negativamente los niveles elevados de ansiedad con el rendimiento, estableciendo a priori que la ansiedad tiene más efectos negativos que positivos en la ejecución de una acción motriz (Prieto, 2016;Ries, Castañeda, Campos\& del Castillo, 2012; Ruiz-Juan, Zarauz \& Flores-Allende, 2015). Un estudio realizado por Ruiz-Pérez, Moreno-Murcia, Ramón-Otero y Alias-García (2015a) con población juvenil, halló que los y las jóvenes no percibían como muy estresantes los contextos de EF. Por su parte, Sola, Martínez, Prados y Martín (2003), hallaron que aquellos sujetos que tenían niveles más altos de ansiedad, percibían las situaciones con mayor riesgo. Así este incremento en la percepción de amenazas hacía que sus decisiones a la hora de realizar una $u$ otra acción se orientaran hacia lo conocido, evitando ciertas situaciones por miedo al fracaso. Es necesaria la adecuación de las sesiones de EF al nivel del alumnado, para que afronte el reto y la mejora con responsabilidad (Moreno, Cervelló, Montero, Vera \& García-Calvo, 2012).

La revisión bibliográfica induce a pensar en la necesidad de conocer la realidad actual las jóvenes en las sesiones de EF y tenerla en consideración. Si bien es cierto que las adolescentes se encuentran en una etapa de cambios anatómicos, cognitivos, motrices, afectivos y sociales que afectan directamente sobre las percepciones que tienen de sí mismas (Del Castillo, 2009; Rodríguez, Mata \& Ruiz, 2006; Vázquez, 2002), son muy importantes los comportamientos asociados al género que se trasmiten en el aula, ya que determinarán su autopercepción y su relación con la práctica de actividad física y deporte. Esta reflexión ha llevado a plantear el presente estudio cuya finalidad es, conocer la competencia motriz real y la ansiedad y el compromiso de las chicas en EF en función de su edad, así como indagar en la relación entre el nivel de competencia motriz, la ansiedad y el compromiso de las jóvenes adolescentes en las sesiones de EF.

\section{Material y Método}

\section{Participantes}

En este estudio participaron 433 chicas de entre 12 y 15 años, siendo la distribución de la muestra según la edad la siguiente: el 29.8\% de 15 años, el $22.4 \%$ de 14 años, el $23.8 \%$ de 13 años y $24 \%$ de 12 años. Todas las jóvenes residían en la Comunidad Autónoma Vasca (Guipúzcoa, Álava y Vizcaya). La técnica de muestreo fue por conveniencia, seleccionando aquellos centros que estaban interesados en participar en el estudio. Con el objetivo de que la muestra estuviese equilibrada, los criterios para la selección de la muestra fueron: territorio histórico, edad y curso escolar, siendo el nivel de confianza del $95 \%$.

\section{Diseño y Variables}

Se trata de un diseño ex post facto de naturaleza descriptiva, comparativa y correlacional de corte transversal.

Las variables analizadas en esta investigación fueron edad, competencia motriz, ansiedad ante el fracaso, y compromiso y entrega en las clases de EF.

La competencia motriz se entiende como la habilidad que tiene la persona para hacer frente a un problema motriz que se le presenta. La ansiedad ante el fracaso hace referencia a «un estado emocional negativo que acompaña una sensación de nerviosismo, preocupación y aprensión, y se relaciona con la activación del organismo» (Weinberg \& Gould, 2010, p.78). Y el compromiso y entrega se refiere al interés e implicación del sujeto hacia las diferentes actividades y tareas planteadas.

En la primera parte del estudio la edad es la variable independiente y el resto actúan como dependientes. En la segunda parte del estudio la competencia motriz actúa como variable independiente y las variables psicosociales actúan como dependientes.

\section{Instrumentos}

Para medir la competencia motriz se adaptó la Batería SPORTCOMP de Ruiz, Graupera, García, Arruza, Palomo y Ramón (2010).Se establecieron 2 variables: coordinación y control motor (saltos laterales, 7 metros saltando a la pata coja, 7 metros saltando con pies juntos, desplazamiento sobre soportes y equilibrio), y aptitud física (lanzamiento de balón medicinal, flexibilidad, dinamometría, carrera ida y vuelta, y abdominales). Para la validación del instrumento se realizó una prueba piloto con 60 chicos y chicas adolescentes y se comprobó la adecuación de las pruebas y el nivel de comprensión de los cuestionarios. Los análisis de fiabilidad fueron de á $=.90$ por lo que se consideró aceptable.

En la tabla1 se resume cada una de las pruebas de la Batería SPORTCOMP:

\begin{tabular}{|c|c|c|}
\hline & Coordinación y control motor & \\
\hline Prueba & Descripción & Puntuación \\
\hline Saltos laterales & $\begin{array}{l}\text { Saltos laterales con los pies juntos sobre un tablero } \\
\text { dividido en la mitad por un listón. Mayor número de } \\
\text { saltos posibles en un tiempo de } 15 \text { segundos. }\end{array}$ & Número de saltos correctos realizados. \\
\hline $\begin{array}{l}7 \mathrm{~m} \text { saltando a la } \\
\text { pata coja }\end{array}$ & $\begin{array}{l}\text { Salto a la pata coja en una distancia de } 7 \text { metros en el } \\
\text { menor tiempo posible. Se atctala pierna preferida. }\end{array}$ & $\begin{array}{l}\text { Tiempo encompletar la tarea (segundos y } \\
\text { decimas de segundo). }\end{array}$ \\
\hline $\begin{array}{l}7 \text { metros saltando } \\
\text { con pies juntos }\end{array}$ & $\begin{array}{l}\text { Salto con pies juntos en una distancia de } 7 \text { metros en el } \\
\text { menor tiempo posible. }\end{array}$ & $\begin{array}{l}\text { Tiempo encompletar la tarea (segundos y } \\
\text { decimas de segundo). }\end{array}$ \\
\hline $\begin{array}{l}\text { Desplazamiento } \\
\text { sobre soportes }\end{array}$ & $\begin{array}{l}\text { Desplazamiento sobre dos soportes en una distancia de } 3 \\
\text { metros en el menor tiempo posible. }\end{array}$ & $\begin{array}{l}\text { Tiempo en completar la prueba } \\
\text { (segundos y décimas de segundo) }\end{array}$ \\
\hline Equilibrio & $\begin{array}{l}\text { Un pie sobre una barra de madera, con las manos en las } \\
\text { caderas y los ojos cerrados, mantenerse en equilibrio sin } \\
\text { ayuda. }\end{array}$ & $\begin{array}{l}\text { Tiempo en equilibrio (segundos y } \\
\text { décimas de segundo) }\end{array}$ \\
\hline & Aptitud física & \\
\hline rueba & Descripción & Puntuación \\
\hline $\begin{array}{l}\text { anzamiento de } \\
\text { 3alón medicinal } \\
\mathrm{kg}\end{array}$ & $\begin{array}{l}\text { Sujetando el balón con las dos manos a la altura del } \\
\text { pecho, proyectarlo lo más lejos posible. }\end{array}$ & Distancia alcanzada en $\mathrm{cm}$. \\
\hline ibilidad & 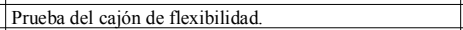 & Distancia alcanzada en $\mathrm{cm}$. \\
\hline & & \\
\hline Carrera ida-vuelta & $\begin{array}{l}\text { En un espacio marcado de } 9 \text { metros, el/la escolar } \\
\text { a la máxima velocidad para recoger el primero de los } \\
\text { relevos colocado en la línea de fondo de } 9 \mathrm{~m} \text { y dejarlo } \\
\text { detrás de la línea de salida. áç ' } \mathrm{G} \text { la misma } \\
\text { operación con un segundo relevo. Una vez colocado la } \\
\text { testigo en el suelo traspasada la línea, finalizado la } \\
\text { prueba. }\end{array}$ & $\begin{array}{l}\text { Tiempo encompletar la distancia } \\
\text { (segundos y decimas de segundo). }\end{array}$ \\
\hline Abdominales & $\begin{array}{l}\text { Tumbados en el suelo, con los brazos cruzados sobe el } \\
\text { pechoy las rodillas flexionadas, incorporarse hasta } 90^{\circ} \text {. }\end{array}$ & Número de repeticiones en 30 segundos \\
\hline
\end{tabular}

En relación a las variables compromiso y ansiedad, se utilizó una adaptación española del Test de Motivación de 
Logro para el aprendizaje en Educación Física, AMPET-R (Ruiz, Graupera, Gutiérrez \& Nishida, 2004). Este cuestionario fue diseñado por Nishida para evaluar la motivación de logro para el aprendizaje en educación física. Más tarde, Ruiz et al. (2004), realizaron una adaptación de este cuestionario al castellano que consta de 37 ítems. En este estudio se aplicó una versión reducida de la versión española del Test AMPET que consta de 33 ítems. Los análisis de fiabilidad de esta versión reducida en castellano muestran un nivel de fiabilidad de á $=.81$. Los tres factores de esta escala muestran los siguientes coeficientes de consistencia interna: compromiso y entrega á $=.81$, competencia motriz percibida á $=$ .92 y ansiedad ante el error y situaciones de estrés á $=.79$. Se realizó una traducción al euskera por el procedimiento de traducción hacia adelante o directa, que consiste en que un grupo de traductores traduce la escala y otro grupo juzga su equivalencia (Carretero-Dios \& Meléndez, 2005). La fiabilidad de la versión traducida al euskera, muestra un nivel aceptable de fiabilidad, con un á $=.83$. La consistencia interna para cada factor fue de á $=.85$ para la competencia motriz percibida, á $=.68$ para el compromiso y entrega, y de á $=.77$ para ansiedad ante el error y situaciones de estrés.

\section{Procedimiento}

El procedimiento para la obtención de datos comenzó con una selección de centros escolares de poblaciones superiores a 4000 habitantes. Tras establecer contacto con los responsables de los centros, se explicaron los objetivos y el procedimiento del proyecto, y posteriormente se obtuvieron los permisos pertinentes. Se concretó el calendario y se procedió a la toma de datos. Una vez en la clase de EF, se realizó una breve explicación y se aclararon las dudas. Seguidamente se procedió a la ejecución del test motor y la cumplimentación de los cuestionarios. La participación fue voluntaria, con una duración total de 2 horas. La categorización de los niveles de competencia motriz hace referencia al estudio realizado en la Comunidad de Trabajo de los Pirineos (Aragón, Barcelona, Andorra y Comunidad Autónoma Vasca) con una muestra de más de 3600 sujetos (Arruza, et al., 2011). En este estudio se establecieron baremos para la variable competencia motriz, en función de edad y sexo, obteniendo tres niveles: alta, media y baja competencia motriz.

Para obtener esta variable previamente se tipificaron los valores de las pruebas que componen el test motor SPORTCOMP. Las pruebas que se medían en tiempo ( $7 \mathrm{me}-$ tros saltando a la pata coja, 7 metros saltando con pies juntos, desplazamientos sobre soportes y carrera ida y vuelta) se multiplicaron por (-1), pues son pruebas de valor inverso: a mayor tiempo peor puntuación. Tras la tipificación de los valores se separaron por un lado las pruebas referentes a las capacidades coordinativas ( 7 metros saltando a la pata coja, 7 metros saltando con pies juntos, desplazamientos sobre soportes y equilibrio) y por otro las pruebas que median las capacidades condicionales (abdominales, dinamometría, lanzamiento de balón medicinal, flexibilidad, carrera ida y vuelta), obteniendo así 2 variables con valores positivos. Para obtener la competencia motriz, se realizaba una media de los valores obtenidos en las 2 variables, coordinativas y condicionales, obteniendo de esta manera un valor entre 33 y 63 .

\section{Análisis estadístico}

Se utilizó el programa estadístico SPSS (Statistical Packagefor the Social Sciencies) para PC versión 22.0.

Dado que las variables siguen una distribución normal se realizaron análisis paramétricos. Se comenzó con análisis descriptivos para las diferentes variables de la muestra según la edad de las participantes. Se utilizaron medidas de tendencia central, para posteriormente realizar comparaciones de medias (ANOVAS) y pruebas post hoc (HSCTukey). Para obtener las relaciones entre la competencia motriz y las variables psicosociales se realizaron correlaciones de Pearson.

\section{Resultados}

Al analizar el nivel de competencia motriz los resultados indicaron que el 37.2\% de las jóvenes tenían un alto nivel. El porcentaje de aquellas que obtuvieron un nivel medio fue de $36.5 \%$, frente al $26.3 \%$ de jóvenes que obtuvieron un bajo nivel de competencia motriz (Figura 1).

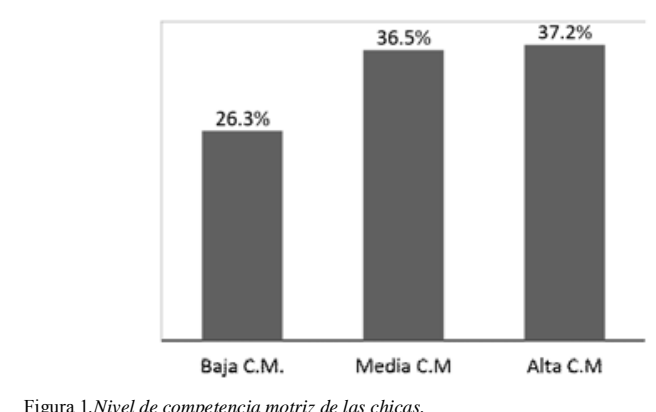

Al estudiar la evolución de la competencia motriz en las chicas se observó que a medida que aumentaba la edad, aumentaba el nivel (tabla 2), siendo este relación positiva y estadísticamente significativa $\left(.31^{* *}\right)$. Con el objetivo de profundizar en esta relación se realizaron análisis ANOVA (tabla 3). Los resultados fueron estadísticamente significativos $F$ (3, 429) $=16.066 ; \mathrm{p}=.000$.

\begin{tabular}{|c|c|c|c|c|c|c|c|c|c|c|c|}
\hline \multirow{2}{*}{\multicolumn{12}{|c|}{ Correlación entre la Competencia Motriz, los facto }} \\
\hline & & & & & & Ansiedad & \multicolumn{2}{|c|}{ Compromiso } & \multicolumn{3}{|c|}{ Edad } \\
\hline \multirow{2}{*}{\multicolumn{3}{|c|}{$\begin{array}{l}\text { Competencia Motriz } \\
\text { Edad }\end{array}$}} & \multirow{2}{*}{\multicolumn{2}{|c|}{$-.14 * *$}} & & & \multicolumn{2}{|c|}{$.16^{* *}$} & \multicolumn{3}{|c|}{$.31 * *$} \\
\hline & & & & & & & $-.20^{*}$ & & & - & \\
\hline \multicolumn{12}{|c|}{$\mathrm{P}<.01 * *$} \\
\hline \\
\hline \multicolumn{12}{|c|}{ Diferencias de medias de C.M., Ansiedad y Compromiso en función de la } \\
\hline \multirow{2}{*}{\multicolumn{3}{|c|}{ M D.T }} & $13 \mathrm{a}$ & & 14 & años & $15 \mathrm{a}$ & años & $F(3,429)$ & & $p$ \\
\hline & & & $\mathrm{M}$ & D.T & $\mathrm{M}$ & D.T & M & D.T & & & \\
\hline C.M. & 41.33 & 3.91 & 42.87 & 4.48 & 44.20 & 3.96 & 44.91 & 4.22 & 16.066 & & .000 \\
\hline Ansiedad & 2.29 & .66 & 2.22 & .65 & 2.36 & .62 & 2.23 & .61 & 1.041 & & .374 \\
\hline Compromiso & 3.06 & .47 & 3.08 & .5 & 2.85 & .5 & 2.82 & .44 & 9.077 & & .000 \\
\hline
\end{tabular}

En la profundización de las diferencias halladas, las pruebas post hoc HSCTukey, señalaron que existían diferencias estadísticamente significativas $(\mathrm{p}=.000)$ entre los grupos de 12 años y el resto de grupos (12-13 años: diferencia de 1.54; entre 12 y 14 años: diferencia de 2.87 ; entre 12 y 15 años: diferencia de 3.58). Sin embargo, el aumento en la media de la competencia motriz en las edades de 13 a 14 años (1.32) y de 14 a 15 años (0.71) no resultó estadísticamente significativo.

En relación a la ansiedad y el estrés de las chicas ante las sesiones de EF, se pudo observar cómo los valores fluctuaron entorno a los 2.25 puntos en función de la edad, alcanzando su máximo nivel a los 14años (ver tabla 3). Cabe seña- 
lar que las diferencias por edad no resultaron estadísticamente significativas.

Respecto a la relación entre la edad y el compromiso y entrega en el aprendizaje, (ver tabla 2), se observó una relación negativa y estadísticamente significativa (-.20**). Las puntuaciones medias obtenidas en esta variable (ver tabla 3), mostraron que las chicas de 13 años obtuvieron las puntuaciones medias más altas $(\mathrm{M}=3.08$ y $\mathrm{Dt}=.50$, en un rango de 1 a 4). A la edad de 14 y 15 años se observó una disminución en las puntuaciones medias $(\mathrm{M}=2.85 ; \mathrm{Dt}=.5 \mathrm{y} \mathrm{M}=2.82$; $\mathrm{Dt}=.44$, respectivamente). Las diferencias halladas entre edades resultaron estadísticamente significativas $F(3$, 429) $=9,077 ; \mathrm{p}=.000$.

Para conocer las diferencias entre los grupos, los análisis post hoc mostraron diferencias estadísticamente significativas, por un lado entre los grupos de 12 y 14 años con una diferencia de 0.16 puntos $(\mathrm{p}=.001)$, entre los grupos de 12 y 15 años con una diferencia de 0.22 puntos $(p=.000)$, y por otro lado, entre los grupos de 13 y 14 años con una diferencia de 0.12 puntos $(\mathrm{p}=.05)$ y entre los grupos de 13 y 15 años con una diferencia de 0.18 puntos $(\mathrm{p}=001)$.

$\mathrm{Al}$ analizar la relación de la competencia motriz con los factores psico-sociales: compromiso y entrega, y ansiedad (ver tabla 2), se observó que a mayor nivel de competencia motriz, las chicas presentaban un mayor compromiso hacia su práctica $(\mathrm{p}<.01)$ y menos ansiedad ante el fracaso $(p<.01)$.Para profundizar en los datos se procedió a realizar análisis ANOVA de un factor. Los datos mostraron (tabla 4) que las jóvenes con baja competencia motriz obtenían puntuaciones medias más altas en ansiedad y menores puntuaciones en compromiso y entrega $(\mathrm{M}=2.39$; $\mathrm{Dt}=.69$ para ansiedad y $\mathrm{M}=2.79 ; \mathrm{Dt}=.54$ para compromiso). Las diferencias entre las puntuaciones medias resultaron estadísticamente significativas para la ansiedad $\mathrm{F}(2,430)=7.173, \mathrm{p}=.001$, y para el compromiso $\mathrm{F}(2,430)=8.750, \mathrm{p}=.000$. Respecto a las diferencias observadas en ansiedad tras las pruebas post hoc (HSDTukey), se encontraron diferencias estadísticamente significativas entre los grupos de baja y alta competencia motriz $(-0.22)$ y media y alta competencia motriz $(-0.19)$ a un nivel de significación de 0.11 . Se procedió a analizar las medias en compromiso y entrega. Las diferencias fueron estadísticamente significativas entre los grupos de baja y alta competencia motriz siendo la diferencia de 0.11 puntos con nivel de significación de 0.05 .

Tabla 4

Diferencias de medias de Ansiedad y Compromiso en función del nivel competencia motriz (rango 1-4)

\begin{tabular}{cccccccccccc}
\hline & \multicolumn{3}{c}{ Baja C.M. } & \multicolumn{4}{c}{ Media C.M. } & \multicolumn{3}{c}{ Alta C.M. } & $F(2$, \\
& $\mathrm{N}$ & M & DT & N & M & DT & N & M & DT & $430)$ & $p$ \\
\hline Ansiedad & 114 & 2.39 & .69 & 158 & 2.34 & .59 & 161 & 2.12 & .61 & 7.173 & .001
\end{tabular}

\begin{tabular}{llllllllllll} 
Compromiso & 114 & 2.79 & .54 & 158 & 2.96 & .47 & 161 & 3.04 & .45 & 8.750 & .000 \\
\hline
\end{tabular}

\section{Discusión}

Este trabajo ha analizado la competencia motriz real y la ansiedad y el compromiso de las chicas en EF en función de su edad, y las relaciones que se establecen entre el nivel de competencia motriz, la ansiedad y el compromiso de las adolescentes en las sesiones de educación física.

Tomando como referencia los baremos establecidos en el estudio de la Comunidad de Trabajo de los Pirineos (Arruza, et al., 2011), se puede decir que los datos obtenidos van en la misma línea pues, las chicas en el presente estudio han obtenido niveles medios y altos de competencia motriz, situándose el 73,7\% de la muestra en dichos parámetros. Se observa que a mayor edad, el nivel de competencia es mayor y esta diferencia es estadísticamente significativa, aunque cabe señalar que a partir de los 14 años se produce una meseta. Estos datos vienen avalados por los obtenidos en otros estudios (Navarro-Valdivielso et al., 2012) en los que se verificaba el descenso de los niveles de práctica a lo largo del segundo ciclo educativo, acentuado en el caso de las chicas. Estos datos podrían apoyarse en diversos estudios que apuntan que los chicos a esta edad experimentan mayor interacción motriz, y son más activos que las chicas (Gallegos-Sánchez, et al., 2019; Lamoneda \& Huerta, 2017), quienes ocupan menos espacios de juego y se vuelven más sedentarias.

Al estudiar los niveles de ansiedad de las chicas en las sesiones de $\mathrm{EF}$, los datos inducen a pensar que las jóvenes se sienten relativamente cómodas ( 2 puntos en un rango de 1-4) a todas las edades. Los resultados obtenidos en la presente investigación son consistentes con los obtenidos en investigaciones previas (Prieto, 2016; Ries, et al., 2012; RuizJuan, et al., 2015), en que las chicas mostraron puntuaciones similares en todas las edades analizadas.

Como se viene observando en la presente investigación, a la edad de 14 años, edad critica en el desarrollo madurativo de las adolescentes, el nivel de ansiedad aumenta ligeramente para posteriormente, a los 15 años, descender. Este descenso puede ser explicado por la maduración cognitiva que se produce en las jóvenes a esta edad y como argumentan distintos autores (Coterón, et al., 2013; Duda \& Hall, 2001) justificaría la disminución de los niveles de ansiedad en la etapa final de la enseñanza secundaria obligatoria. Las participantes en esta investigación no muestran en ningún caso puntuaciones altas en ansiedad, lo que indica que no perciben como estresantes los contextos de EF (Ruiz-Pérez, at al., 2015a). No obstante, las jóvenes con un nivel de competencia motriz alto, perciben menos ansiedad que las de nivel medio y estas menos que las de nivel bajo. Resultaría interesante atender los hallazgos del estudio realizado por Granado-Gallegos y Baena-Extremera (2014) que muestra que las chicas se sienten más cómodas en un clima de aprendizaje.

Respecto a los datos obtenidos sobre el compromiso y entrega en las clases de EF, las chicas presentan valores medios, es decir, están comprometidas con las sesiones sin embargo, este compromiso desciende a medida que se hacen mayores, siendo estas diferencias significativas, y este problema se focaliza en las chicas a partir de los 14 años. En el compromiso deportivo, tal y como apuntan Moreno y Cervelló (2010) es importante estar motivado intrínsecamente, es decir, practicar por el propio disfrute de la actividad, lo que lleva a pensar, en concordancia con distintos autores (Fontecha, 2006; Sever, 2005), que las tareas planteadas en las sesiones de EF no son atractivas para las jóvenes de más edad. Autores como Yoo (2015) apuntan la motivación autónoma como mediadora de la competencia percibida y del compromiso conductual. Además, se constata que a mayor nivel de competencia, la intención de seguir practicando actividad físico-deportiva es mayor (Arribas-Galarraga, et a 1., 2018), por tanto, como indican Stuntz y Weiss (2009), sería 
necesario estudiar los factores que influyen sobre su diversión y su sentimiento de competencia, para evitar una falta de motivación hacia la asignatura.

Finalmente, el presente estudio confirma que el nivel de competencia motriz de las adolescentes se relaciona negativa y significativamente con los niveles de ansiedad, y positiva y significativamente con el compromiso en el aprendizaje, es decir, que a medida que aumenta el nivel de competencia disminuye la ansiedad y mejora la implicación en las clases de EF. Este dato está en línea con los resultados obtenidos por diferentes autores (Lima, et al., 2015; Prieto, 2016), quienes confirman que la mejora de la competencia motriz constituye un aspecto importante en la motivación, determinante del comportamiento humano y elemento clave para logran adherencia y compromiso en una actividad. Desde un punto de vista teórico, esta influencia se podría explicar con la teoría de la autodeterminación propuesta por Deci y Ryan (1985), que se estructura en un continuo que integra diferentes niveles de motivación, desde la amotivación hasta la motivación intrínseca, siendo esta la más autodeterminada y asociada al disfrute por la ejecución de la tarea y el compromiso (Torregrosa et al. 2008; Torregrosa et al. 2011).

Por todo ello se puede considerar que un alto nivel de competencia motriz favorece el aumento del compromiso con la actividad y disminuye los niveles de ansiedad, y que esto repercute positivamente en la mejora de la propia competencia motriz. En esta línea coincidimos con autores como Moreno, et al. (2012) quienes destacan la necesidad de que las sesiones de EF y las tareas a desarrollar estén planteadas y sean asequibles al nivel de competencia del alumnado, para que así puedan afrontar su mejora y aceptar su responsabilidad, midiendo su propio progreso. En este sentido (MéndezGiménez et al. 2013), un clima tarea en el que se asocian la orientación de meta y los niveles positivos de motivación intrínseca, obtendrá altos niveles en compromiso y bajos niveles de ansiedad y estrés.

Esta investigación aporta nueva información en lo concerniente al nivel de competencia motriz real y su relación con la ansiedad y compromiso con la EF curricular. A nivel general, la edad de las participantes se muestra como variable que marca una tendencia en el descenso del compromiso con las clases de EF, mientras que la ansiedad fluctúa y no resulta significativa. Los 14 años se presentan como edad crítica para las chicas por lo que sería conveniente realizar más estudios en los que desde un clima de aprendizaje se abordaran contenidos que pudieran generar percepción de competencia. Por otro lado, este estudio constata la competencia motriz como eje del alto compromiso y menor ansiedad durante las sesiones, independientemente de la edad; es decir, las chicas más competentes, independientemente de su edad, resultan ser las más comprometidas con las sesiones de EF, y a su vez son las que menores niveles de ansiedad presentan.

Finalmente, el trabajo expuesto induce a pensar en la necesidad de abordar las propuestas en EF para las jóvenes a partir de los 14 años. Posiblemente el planteamiento de actividades atractivas para las chicas, con adecuados niveles de competencia, podrían incrementar el compromiso promoviendo actitudes más activas en las chicas.

\section{Agradecimientos}

Expresamos nuestro sincero agradecimiento a todas las personas y entidades que han hecho posible este estudio: Departamento de Educación, Política Lingüística y Cultura del Gobierno Vasco (CTP09-P02 y Ref: BFI09.215), y centros educativos que han participado (Gipuzkoa: Zurriola Ikastola, TokiAlai Ikastola, Arizmendi Ikastola, Urretxu-Zumarraga Ikastola, San Jose Ikastetxea, El Pilar Ikastetxea; Bizkaia: San Felix de Cantalicio Ikastetxea, Jado Ikastetxea, Lauaxeta Ikastola, Astileku Ikastola, San Antonio Santa Rita Ikastetxea; Araba: Ikasbidea Ikastola, UrkideIkastetxea, IES Mendebaldea BHI, IES Francisco de Vitora BHI).

\section{Referencias}

Ames, C. (1987). The enhancement of student motivation. En D. Kleiber y M. Maehr (Ed.), Advances in motivation and achievement (pp. 123-148) Greenwich, CT: JAI Press.

Arguedas, I. (2010). Involucramiento de los estudiantes y los estudiantes en el proceso educativo. Revista Electrónica Iberoamericana sobre Calidad, Eficacia y Cambio en Educación, 8 (1), 63-78.

Ariolfo, R. (2010). La dimensión socio-afectiva: un factor clave para el aprendizaje y la integración de los jóvenes de Latinoamérica en el contexto escolar genovés. European Information Network on International Relations and Area Studies (INIRAS), 95-105.

Arribas-Galarraga, S., Luis-de Cos, G., Luis-de Cos, I., \& Saies, E. (2018). Young girls: motor competente, sport practice and intention of future practice. Journal of Sport and Health Researh, 10, 135-144.

Arruza, J. A., Irazusta, S., \& Urrutia-Gutierrez, S. (2011). Evaluación de la competencia motriz en los escolares de la educación segundaria obligatoria de las regiones de la Comunidad de Trabajo de los Pirineos. (CTP09-18). Donostia: Gobierno Vasco.

Astin, A. W. (1999). Student Involvement: A Developmental Theory for Higher Education. Journal of College Student Development, 40 (5), 518529.

Cairney,J., Kwan,M., Velduizen,S. , Hay.J., Bray,S.R., \&Faught,B.E. (2012) Gender, perceived Competence and the enjoyment of physical education in children: a longitudinal examination. International Journal of Behavioral Nutrition and Physical Activity9 (26), 1-8.

Cantó, E. G., García, P. L. R., Soto, J. J. P., Guillamon, A. R., \&Villalba, F. J. L. (2015). Autopercepción de competencia motriz y su relación con la práctica físico-deportiva en escolares de la Región de Murcia (España).REXE: Revista de estudios y experiencias en educación, 14(27), 49-61

Carretero-Dios, H.,\& Pérez, C. (2005). Normas para el desarrollo y revisión de estudios instrumentales. Internacional Journal of Clinical and Health Psychology, 5, 521-551.

Cecchini, J. A., González, C., López, J., \& Brustad, R. J. (2005).Relación del clima motivacional percibido con la orientación de meta, la motivación intrínseca y las opiniones y conductas de fairplay. Revista Mexicana de Psicología, 22(2), 469-479. 
Cera, E.,Almagro, B.J., Conde, C.,\& Sáenz-López, P. (2015).Inteligencia emocional y motivación en educación física en secundaria. Retos. Nuevas tendencias en Educación Física, Deporte y Recreación, 27, 8-13.

Connolly, K. (1980). The development of motor competence.En C.H. Nadeau y cols. (Ed). Psycology of motor behavior and sport-1979.Champaing, Human Kinetics.

Connolly, K.,\& Bruner, J. (1973).The growth of competence.London: AcademicPress.

Coterón, J., Franco, E., Pérez-Tejero, J., \& Sampedro, J. (2013). Clima motivacional, competencia percibida, compromiso y ansiedad en Educación Física. Diferencias en función de la obligatoriedad de la enseñanza. Revista de Psicología del Deporte, 22 (1), 151-157.

Deci, E. L., \& Ryan, R. M. (1985).Intrinsic motivation and self-determination in human behavior.Nueva York: PlenumPress.

Del Castillo, O. (2009). Evaluación de los factores psicosociales y didácticos relacionados con la equidad de género en educación física. (Tesis doctoral inédita). Sevilla: Universidad de Sevilla.

Duda, J. L., \& Hall, H. (2001). Achievement goal theory in sport: Recent extensions and future directions. En R. N. Singer, H. A. Hausenblas, y C. M. Janelle (Eds.), Handbook of sport psychology (pp. 417-443). New York: Wiley

Fontecha, M. (2006). Intervención didáctica desde la perspectiva de género en la formación inicial de un grupo de docentes de educación física. (Tesis doctoral). Leioa: Universidad del País Vasco/ Euskal Herriko Unibertsitatea.

Fredriscks, J., Blumenfeld, O., \& Paris, A. (2004). School Engagement: Potential of the concept state of the evidence. Review of Educational Research, 74 (1), 59109.

Gallegos-Sánchez, J. J., Ruiz-Juan, F., Villarreal-Angeles, M. A., \& Rivera, J. I. Z. (2019).Etapas de cambio en la práctica de actividad física de tiempo libre en estudiantes de secundaria de Victoria de Durango, México. Retos, (35), 196-200.

Granado-Gallegos, A.,\& Baena-Extremera, A.(2014). Predicción de la motivación autodeterminada según las orientaciones de meta y el clima motivacional en Educación Física. Retos: nuevas tendencias en educación física, deporte y recreación, (25), 23-27

Gutiérrez, M(2014). Relaciones entre clima motivacional, las experiencias en educación física y la motivación intrínseca de los alumnos. Retos. Nuevas tendencias en Educación Física, Deporte y Recreación, 26, 9-14.

Hellì̀n, P., Moreno, J. A., \& Rodríguez, P. L. (2006). Relación de la competencia motriz percibida con la práctica físicodeportiva. Revista de Psicología del Deporte. 5(2), 219231

Lamoneda, J., \& Huerta, F. J. (2017). Análisis de la práctica deportiva recreativa, tipo de interacción motriz, uso del espacio e instalación en adolescentes en función del género. Retos, 33, 30-34.

Lima, F., Saavedra, F., Fernandes, H. M., Lazuras, L., \& Barkoukis, V. (2015). Versión portuguesa de la Escala de Estado de Ansiedad en Educación Física: Propiedades psicométricas y su asociación con el sexo, edad y actividad física extracurricular. Cuadernos de Psicología del Deporte, 15 (3), 135-144.

Méndez-Giménez,A., Fernández-Rio, J., \& Cecchini-Estrada, J.A, (2013). Climas motivacionales, necesidades, motivación y resultados en Educación Física. Aula Abierta, 41 (1), 63-72.

Moreno, J.A., \&Cervelló, E. (2010). Motivación en la actividad física y el deporte. Sevilla. Wanceulen.

Moreno, J. A., Cervelló, E., Montero, Vera, J. A., \& GarcíaCalvo, T. (2012) Metas sociales psicosociales básicas y motivación intrínseca como predictores de la percepción del esfuerzo en las clases de educación física, Revista de Psicología del Deporte, 21, 2, pp. 7-13.

Morente, H., Zagalaz, M. L., Molero, D., \& Carrillo, S. (2012). Prevención de la obesidad infantil a través de una motivación intrínseca hacia la práctica de actividad física. Retos. Nuevas tendencias en Educación Física, Deporte y Recreación, 22, 33-37.

Navarro-Valdivielso, M., Ojeda-García, R., NavarroHernández, M., López-López, E., Brito-Ojeda, E., \& RuízCaballero, J. A. (2012). Compromiso de los adolescentes de Canarias con un estilo de vida físicamente activo y saludable. Retos: nuevas tendencias en educación física, deporte y recreación, 21, 30-33.

Pelegrín, A., León, J. M., Ortega, E., \& Garcés de Los Fayos, E. J. (2012). Programa para el desarrollo de actitudes de igualdad de género en clases de educación física en escolares. Educación XXI, 15 (2).

Prieto, J. M. (2016). Relación entre competitividad, ansiedad social y compromiso con variables deportivas y académicas en futbolistas jóvenes. Revista Iberoamericana de Psicología del Ejercicio y el Deporte, 11 (2), 193-200.

Ries, F., Castañeda, C., Campos, M. C., \& Del Castillo, O. (2012). Relaciones entre ansiedad-rasgo y ansiedad-estado en competiciones deportivas. Cuadernos de Psicología del Deporte, 12 (2), 9-16.

Rigo, D. (2014). Enseñar y aprender. Promoción del compromiso, diseña instructivo e inteligencias múltiples. En J. I. Alonso y R. N. Martínez-Artero, (Eds.), Investigación educativa en educación primaria (pp. 320-336). Universidad de Murcia: Edit. um.

Rigo, D., \& Donolo, D. (2014). Entre pupitres y pizarrones. Retos en educación primaria: el aprendizaje con compromiso. Education Siglo XXI, 32 (2), 59-80.

Rodriguez, M. F., Mata, E., \& Ruiz, L. M.(Diciembre, 2006) El autoconcepto físico en la adolescencia temprana: influencias del género y la edad. Actas del I Congreso de Jóvenes Investigadores en Ciencias de la Actividad Física y el Deporte (pp. 163-172). Valladolid.

Ruiz-Juan, F., Zarauz, A., \& Flores-Allende, G. (2015). Variables predictoras de la ansiedad precompetitiva: aspectos diferenciales en corredores de fondo en ruta. Universitas Psychologica, 14(3), 1021-1032.

Ruiz Pérez, L. M. (1995). Competencia motriz. Elementos para comprender el aprendizaje motor en educación física escolar. Madrid, Gymnos.

Ruiz, L.M. (2014). De qué hablamos cuando hablamos de competencia motriz. Acción motriz, 12, 37-44.

Ruiz, L.M., \& Arruza, J. A. (2005). El proceso de toma de 
decisiones en el deporte. Clave de la eficiencia y el rendimiento óptimo. Barcelona: Paidos Ibérica.

Ruiz, L.M., \& Graupera, J.L. (2005). New measure of perceived motor competence for children age 4 to 6 years.Perceptual and Motor, 101, 131-148.

Ruíz, L. M., Graupera, J. L, García, V., Arruza, J. A., Palomo, M., \& Ramón, I. (2010). Batería Multidimensional de la Competencia Motriz-Sportcomp. Toledo: Laboratorio de Competencia Motriz. Universidad de Castilla la Mancha.

Ruíz, L. M., Graupera, J. L., Gutiérrez, M., \&Nishida, T. (2004). El test de motivación de logro para el aprendizaje en educación física: Desarrollo y análisis factorial de la versión española. Revista de Educación, 335, 195-211.

Ruiz, L.M., \& Linaza, J.L. (2013). Jerome Brunery la organización de las habilidades motrices en la infancia. Revista Internacional de Ciencias del Deporte (RICYDE), 34, 390-397.

Ruiz-Pérez, L. M., Moreno-Murcia, J. A., Ramón-Otero, I., \& Alias-García,A. (2015a). Motivación de Logro para Aprender en Educación Física: Adaptación de la versión española del Test AMPET. Revista Española de Pedagogía, 260, 157-175.

Ruiz-Pérez, L. M., Rioja-Collado, N., Graupera-Sanz, J. L., Palomo-Nieto, M., \& García-Gil, V. (2015b). GRAMI-2: Desarrollo de un test para evaluar la coordinación motriz global en la educación primaria. Revista iberoamericana de psicología del ejercicio y el deporte, 10 (1), 103 111.

Sever, C. (2005). Genero \& deporte: Integrar la equidad de género en los proyectos deportivos. COSUDE, Agencia Suiza para el Desarrollo y la Cooperación.

Sola, R., Martínez, A.R., Prados, A., \& Martin, J. (2003). La ansiedad de los individuos en la sociedad del riesgo. Ansiedad y Estrés, 9(1), 93-103.

Smith, R. E., Smoll, F. L., \& Cumming, S. P. (2007). Effects of a motivational climate intervention for coaches on young athletes' sport performance anxiety. Journal of Sport and Exercise Psychology, 29, 39-59.

Stuntz, C. P., \& Weiss, M. R. (2009). Achievement goal orientations and motivational outcomes in youth sport: The role of social orientations. Psychology of Sport and Exercise 10, 255-262.

Torregrosa, M., Sousa, C., Viladrich, C., Villamarín, F., \& Cruz, J. (2008).El clima motivacional y el estilo de comunicación del entrenador como predictores del compromiso en futbolistas jóvenes. Psicothema, 20(2), 254-259.(

Torregrosa, M., Viladrich, C., Ramis, Y., Azócar, F., Latinjak, A. T., \& Cruz, J. (2011). Efectos en la percepción del clima motivacional generado por los entrenadores y compañeros sobre la diversión y el compromiso. Diferencias en función del género. Revista de Psicología del Deporte, 20(1), 243-255.

Urrutia-Gutierrez, S., Irazusta-Adarraga, S., Balerdi-Iztueta, A., González-Rodríguez, O, \& Arruza-Gabilondo, J. A. (2013). Relación entre el índice de masa corporal y la competencia percibida en adolescentes de la Educación Secundaria Obligatoria. Retos: nuevas tendencias en educación física, deporte y recreación, (24), 21-23.

Vázquez, B. (2002). Los valores corporales y la Educación Física. Trabajo presentado en el II congreso de la asociación Española de Ciencias del Deporte, marzo, Madrid.

Weinberg, R. S., \& Gould, D. (2010).Fundamentos de Psicología del Deporte y del Ejercicio físico. Madrid: Panamericana

White, R.W. (1959). Motivation reconsidered: The concept of competence. PsycologyReview, 66 (5), 297-323.

Yoo, J. (2015). Perceived autonomy support and behavioral engagement in physical education: A conditional process model of positive emotion and autonomous motivation. Perceptual and motor skills, 120(3), 731-746.

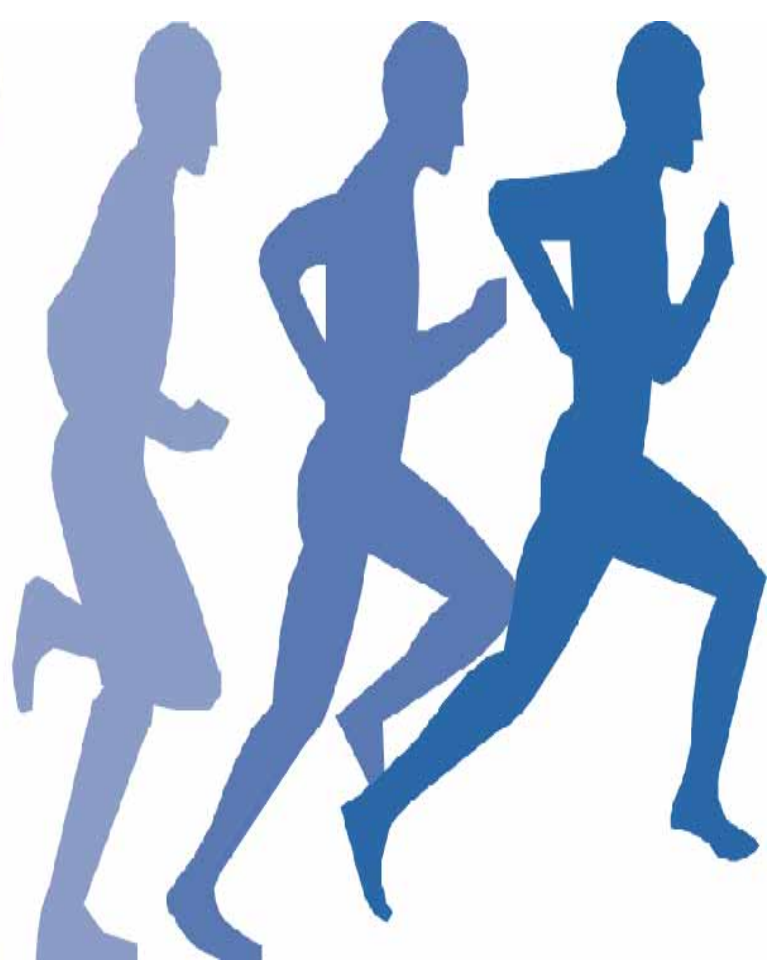

